\title{
Cercas vivas en sistemas de producción tropicales: una revisión mundial de los usos y percepciones
}

\author{
Javier Leonardo Morantes-Toloza ${ }^{1 *}$ \& Luis Miguel Renjifo ${ }^{2}$ \\ 1. Maestría de Conservación y Uso de Biodiversidad, Facultad de Estudios Ambientales y Rurales, Pontificia \\ Universidad Javeriana, Bogotá, Colombia; jmorantes@javeriana.edu.co \\ 2. Departamento de Ecología y Territorio, Facultad de Estudios Ambientales y Rurales, Pontificia Universidad Javeriana, \\ Bogotá, Colombia; Imrenjifo@javeriana.edu.co \\ * Correspondencia
}

Recibido 11-VII-2017. C Corregido 16-I-2018. Aceptado 14-II-2018.

\begin{abstract}
Live fences in tropical production systems: a global review of uses and perceptions. The expansion of agricultural and livestock production has been a key factor in deforestation in the world and especially in the tropical region. Currently, more than half of the Earth's surface is used for the establishment of production systems; in this context, live fences can generate positive effects on the environment and provide environmental goods and services. In this work, we reviewed the effects of live fences on production systems from the tropical regions of America, Africa, Asia and Australia, analyzing the uses and perceptions of farmers that have made use of these fences. It was found that live fences were mainly related to the delimitation of farms and pastures. However, their uses and benefits are manifold, among which we found: provide forage, wood extraction, fruit supply, windbreaks, medicinal, to avoid soil erosion, moisture retention and nutritional support. In addition, other positive perceptions were: increasing landscape connectivity, increasing economic incomes, reducing production costs, reducing pressure on remaining vegetation, keeping maintenance costs down, contributing to pest control, and improving fertility. However, negative perceptions included the excessive work in repairing or pruning them, shade negative impact on agricultural production, lack of information discourages establishment, high establishment costs, generation of property conflicts and management difficulty. It was found that the uses by farmers are focused on improving the yields of productive systems, but also on the conservation of natural resources especially soil and biodiversity. We proposed research topics for the future, as determining the criteria in the selection of native species to establish and enrich living fences valuing ecological and socioeconomic aspects; in additionally, we must deepen the benefits they produce on the yields of productive systems. We suggest the development of live fences studies in the tropical region of Australasia, because no data was found for this region, in order to know the biodiversity and services status that they provide to the rural communities. As a conclusion, it will be important to generate initiatives that encourage the formulation of rural policies, the creation of programs that encourage the establishment of live fences, whether through payments for environmental services, tax compensation or other mechanisms. Providing adequate information to farmers and livestock farmers is a key aspect on dynamizing the establishment of live fences. Rev. Biol. Trop. 66(2): 739-753. Epub 2018 June 01.
\end{abstract}

Key words: live fences; tropical biodiversity; silvopastoral systems; agricultural systems; agroforestry.

La expansión de la producción agrícola y ganadera ha sido un factor determinante en la deforestación en el mundo (3.3 ha-2010-2015) $\mathrm{y}$ en las regiones tropicales, especialmente en América del Sur y en África, donde el área de bosque promedio per cápita pasó de 0.8 ha a 0.6 ha por persona entre 1990 y 2015 (Barbier
\& Burgess, 2001; Pereira et al., 2010; Etongo et al., 2015; FAO, 2016). En consecuencia, más de la mitad de la superficie terrestre del planeta se utiliza para la agricultura y ganadería, lo que origina consecuencias en el paisaje, alterando la calidad y conectividad del hábitat (Foley et al 2005, Haddad et al., 2015). Esta presión 
por aumentar la frontera agrícola y elevar la producción de alimentos para una población creciente $(1.19 \%$ - 2016) ha traído como consecuencia, aumentos en el uso de agroquímicos, en la erosión de los suelos, y en el deterioro de las cuencas y fuentes de agua (Murgueitio, Calle, Uribe, Calle, \& Solorio, 2011). En este contexto, los sistemas silvopastoriles han sido propuestos para gestionar los sistemas productivos para mejorar tanto la productividad como la conservación de los recursos naturales (Cubbage et al., 2012, Calle, Murgueitio, \& Chará, 2012). Por ejemplo, se estima que los sistemas agrícolas y ganaderos que integran árboles y altas densidades de arbustos, tienen una dependencia menor a insumos agroquímicos, que reduciría la necesidad de comprarlos masivamente (Garner \& Steinberger, 1989).

Existen diversas combinaciones de plantas leñosas con pasturas herbáceas y animales, lo que da lugar a diferentes tipos de sistemas silvopastoriles, y entre las opciones que presentan mayor frecuencia en la región tropical son las cercas vivas, que con frecuencia se usan para demarcar los campos agrícolas, las pasturas y los límites de las fincas y forman elaboradas redes de cobertura arbórea a lo largo de los paisajes rurales (Burel, 1996; Kinama, Stigter, Ong, Ng'ang'a, \& Gichuki, 2005; León \& Harvey, 2006; Wehling \& Diekmann, 2009; Villanueva-López, Martínez-Zurimendi, Ramírez-Avilés, Casnova-Lugo, \& Jarquín-Sánchez, 2014; Abas, Corpuz, \& Cabilo, 2015).

Los sistemas productivos con cercas vivas son una estrategia de manejo del paisaje rural, la cual no solo contribuye a la recuperación de la diversidad biológica sino también al aumento de la productividad y los rendimientos económicos de los sistemas productivos (Murgueitio, Cuartas, \& Naranjo, 2008).

La presencia de cercas vivas se produce en áreas rurales con distintas culturas, historias de uso del suelo y producción agrícola, sobre todo en regiones donde la deforestación y la conversión del suelo es de manera frecuente (Harvey et al., 2003; Harvey et al., 2005). Además, son una alternativa económica al establecimiento de postes muertos de madera y se han considerado una opción para promover la conservación en paisajes agrícolas y como conexiones físicas entre fragmentos de bosques en paisajes trasformados por la actividad humana (León \& Harvey, 2006; Otero \& Onaindia, 2009; Pulido-Santacruz \& Renjifo, 2011; Wehling \& Diekmann, 2009). Por otro lado, existen percepciones negativas en cuanto al uso de cercas vivas, ya que la falta de apoyo institucional y el alto costo de establecimiento hacen que sea una limitación para su implementación (Pérez et al., 2011).

Desde el punto de vista socioeconómico, los agricultores y propietarios de tierras son quienes toman las decisiones sobre el establecimiento, manejo y uso que hacen de las cercas vivas (Harvey, Tucker, \& Estrada, 2004). Éstas podrían contribuir en la reducción de la explotación de los bosques remanentes, debido a que proveen recursos como madera, leña y postes para construcción, que hace que disminuya la tala sobre los parches de bosques adyacentes (Budowski, 1993; Nascimento, Sousa, Alves, Araújo, \& Albuquerque, 2009; Otero \& Onaindia, 2009; Hermuche, Vieira, \& Sano, 2013).

A pesar de la preeminencia de las cercas vivas en algunos paisajes tropicales, se deben tomar medias para estimular y conservar las cercas vivas. Éstas podrían ser preservadas incluso desde el ámbito legal, y su indagación a nivel socioeconómico en la región tropical, es fundamental para incentivar el establecimiento de estas estructuras arbóreas de manera autosostenible y eficiente en los sistemas productivos tropicales (de Castro \& van den Berg, 2013). El propósito de esta revisión fue determinar los usos que le dan los pobladores locales a las cercas vivas en los paisajes rurales tropicales, e identificar las percepciones, tanto positivas como negativas que hayan sido reportadas en la literatura.

Lo que se espera con la realización de esta revisión de literatura científica, es que las cercas vivas se constituyan en una práctica agroforestal que genere múltiples beneficios a los agricultores, que se pueda fomentar su implementación en la región rural tropical, y que las percepciones sobre ellas sean a favor 
de su utilización y aprovechamiento en los sistemas productivos. Esta constituye la primera revisión de literatura científica para la región tropical.

\section{MATERIALES Y MÉTODOS}

Para la realización de este estudio, se analizaron los artículos publicados en revistas científicas sobre las cercas vivas en las regiones tropicales del mundo (desde los $23^{\circ}$ latitud norte hasta los $23^{\circ}$ latitud sur), en el período comprendido entre 1990 y 2016; por lo tanto, artículos técnicos no fueron incluídos en esta revisión de literatura. Los artículos fueron buscados en Scopus, Web of Science, Scielo, Google Scholar, y se complementó al detallar las referencias de los artículos analizados. La búsqueda se hizo desde el 1 diciembre 2015 hasta el 19 de agosto 2016. Se utilizó como criterio de selección aquellos que tuvieran una relación directa con el uso y percepción de las cercas vivas en sistemas agrícolas y ganaderos en la región tropical. Para la búsqueda bibliográfica se usaron palabras claves en tres idiomas (inglés, español y portugués): cercas vivas, living fences, hedgerow, fencerows, live fence, forest fence, vegetation fence y silvopastoral systems. Estas palabras claves fueron buscadas en el título, el resumen y las palabras claves de cada artículo.

La frase de búsqueda que se utilizó en Scopus fue TITLE-ABS-KEY ("cercas vivas" OR "living fences" OR hedgerow OR fencerows OR "Forest fence" OR "Vegetation fence") AND NOT TITLE-ABS-KEY (Isolated tres OR "Biological corridor"), arrojando 1012 artículos y la sentencia utilizada en Web of Science fue TS=("Living fence*" OR hedgerow OR fencerow OR "live fence*" OR "forest fence" OR "vegetation fence") AND TS=(biodiversity OR species) NOT TS=(Isolated tres OR "Biological corridor"). Para los buscadores Scielo y Google Scholar se dio inicio a la búsqueda de cada palabra clave una a una. Los archivos fueron descargados para su posterior análisis.

\section{RESULTADOS}

Se encontraron un total de 1123 artículos de los cuales se seleccionaron 98 que tenían como eje central las cercas vivas y otros sistemas silvopastoriles. De estos últimos se seleccionaron para el análisis 57 artículos, dado que, en los artículos restantes, no se proveía información pertinente para este estudio. El $70 \%$ de los artículos encontrados tenían información relevante hacia los usos de las cercas vivas por parte de pobladores locales (Cuadro 1) y el 42 $\%$ presentaba información sobre percepciones sobre las cercas vivas (Cuadro 2).

La región donde más se produjeron artículos fue la región neotropical con 40 publicaciones, seguida de la región tropical de África con 12 y Asia tropical con cinco artículos. No se encontraron estudios sobre cercas vivas en Australasia tropical. En general, los países que registraron mayor número de publicaciones fueron México, Costa Rica y Brasil, con valores de once (11), nueve (9) y cinco (5) artículos, respectivamente (Fig. 1).

\section{Usos que le dan los pobladores locales a las cercas vivas en los paisajes rurales tropicales}

El principal uso que le dan a las cercas vivas en la región tropical es para delimitar los sistemas agropecuarios y delimitar los predios, se encontró que las cercas vivas son utilizadas en una amplia variedad de usos (Cárdenas, Harvey, Ibrahim, \& Finegan, 2003; León \& Harvey, 2006; Zuria, Gates, \& Castellanos, 2007; Love, Bork, \& Spaner, 2009; Ruiz-Guerra, López-Acosta, Zaldivar-Riverón, \& VelázquezRosas, 2015) que se describen y clasifican de acuerdo con el servicio que proporcionan.

Provisión de forraje: Del total de artículos, se encontró que un $14.03 \%$ de los artículos en el Neotrópico y un $4 \%$ del trópico africano lo reportaron como para provisión de forraje. La región tropical asiática no reporta el uso mediante literatura científica, pero al parecer 
CUADRO 1

Usos de las cercas vivas por parte de agricultores y ganaderos en la región tropical

TABLE 1

Uses of live fences by farmers and Livestock farmers in the tropical region

\begin{tabular}{|c|c|c|c|}
\hline Uso identificado & Países & Región & Fuente \\
\hline Forraje & $\begin{array}{l}\text { Burkina Faso, Costa } \\
\text { Rica, Cuba, Nicaragua, } \\
\text { México, Kenia }\end{array}$ & $\begin{array}{l}\text { Neotrópico y África } \\
\text { tropical }\end{array}$ & $\begin{array}{l}\text { Somarriba, 1995; Ayuk, 1997; Kiepe, } \\
\text { 1996; Alonso et al., 2002a; Alonso et al., } \\
\text { 2002b; Reyes \& Rosado 2000; Harvey et } \\
\text { al., 2003; Harvey et al., 2005; Kinama et } \\
\text { al., 2005; Love et al., 2009; Siles et al., } \\
\text { 2013; Fuentealba \& Martínez-Ramos, } \\
\text { 2014 }\end{array}$ \\
\hline Madera/Leña & $\begin{array}{l}\text { Burkina Faso, Brasil, } \\
\text { Camerún, Costa Rica, } \\
\text { Honduras, Panamá, } \\
\text { México, Nicaragua, } \\
\text { Senegal }\end{array}$ & $\begin{array}{l}\text { Neotrópico y África } \\
\text { tropical }\end{array}$ & $\begin{array}{l}\text { Romero et al., 1991; Ayuk, 1997; Rowe et } \\
\text { al., 2001; Betancourt et al., 2003; Muñoz } \\
\text { et al., 2003; Villacis et al., 2003; Harvey } \\
\text { et al., 2005; Zahawi, 2005; Love et al., } \\
\text { 2009; Nascimento et al., 2009; Otero \& } \\
\text { Onaindia, 2009; Chavarría et al., 2011; } \\
\text { Pérez et al., 2011; Siles et al., 2013; Ruiz- } \\
\text { Guerra et al., 2014; Sousa et al., } 2016\end{array}$ \\
\hline Provisión de frutos & $\begin{array}{l}\text { Brasil, Costa Rica, } \\
\text { Cuba, Ghana, } \\
\text { Honduras, Indonesia, } \\
\text { Kenia, México, } \\
\text { Nicaragua, Nigeria, } \\
\text { Senegal }\end{array}$ & $\begin{array}{l}\text { Neotrópico, África } \\
\text { tropical y Asia } \\
\text { tropical }\end{array}$ & $\begin{array}{l}\text { Ayuk, 1997; Olasantan, 2000; Reyes } \\
\text { \& Rosado, 2000; Rowe et al., 2001; } \\
\text { Harvey et al., 2003; Muñoz et al., 2003; } \\
\text { Abunyewa et al., 2004; Restrepo-Sáenz et } \\
\text { al., 2004; Kinama et al., 2005; Chavarría } \\
\text { et al., 2011; Fogel et al., 2012; Grechi et } \\
\text { al., 2013; Loyola et al., 2015; }\end{array}$ \\
\hline Productos medicinales & Brasil, Mali & $\begin{array}{l}\text { Neotrópico y África } \\
\text { tropical }\end{array}$ & $\begin{array}{l}\text { Nascimento et al., } 2009 \text {; } \\
\text { Levasseur et al., } 2004\end{array}$ \\
\hline $\begin{array}{l}\text { Evitar la erosión y retener } \\
\text { la humedad del suelo }\end{array}$ & Colombia, México & Neotrópico & $\begin{array}{l}\text { Otero \& Onaindia, 2009; Oshunsanya, } \\
2013 \text { Villanueva-López et al., } 2016\end{array}$ \\
\hline Proporcionar sombra & Cuba & Neotrópico & Fudora et al., 2004 \\
\hline $\begin{array}{l}\text { Aporte de nutrientes } \\
\text { a los cultivos }\end{array}$ & Filipinas, Sri Lanka & Asia tropical & Weerasinghe, 2007; Agus et al., 1999 \\
\hline Rompevientos & India & Asia tropical & Adhikary et al., 2016 \\
\hline
\end{tabular}

las personas en Asia emplean las cercas vivas para proporcionar forraje a sus animales de corral (Horne \& Stür, 1999). Sin embargo, algunos autores reportan que las cercas vivas se usan para suministrar alimento al ganado, sobre todo en época de sequía (Cuadro 1). Las cercas con este fin están compuestas de especies como Gliricidia sepium, Bursera simaruba, Leucaena leucocephala y Erythrina poeppigiana, las cuales suministran forraje de alta calidad nutricional, principalmente cuando el pasto es escaso (Somarriba, 1995; Kiepe, 1996; Ayuk, 1997; Alonso, Febles, Ruiz, Torres, \& Gutiérrez, 2002a; Kinama et al., 2005; Love et al., 2009; Siles, Rayo, Rugama, \& Molina, 2013; Fuentealba \& Martínez-Ramos, 2014). Para proveer forraje al ganado se hacen podas esporádicas dejando el material disponible en el suelo del pastizal, para luego ser consumido por el ganado con mayor facilidad (Alonso, Febles, 
CUADRO 2

Percepciones tanto positivas como negativas de las cercas vivas en los paisajes rurales tropicales

TABLE 2

Positive and negative perceptions of live fences in tropical rural landscapes

\begin{tabular}{|c|c|c|c|}
\hline Percepción identificada & Explicación & País & Fuente \\
\hline \multicolumn{4}{|c|}{ Percepciones positivas } \\
\hline $\begin{array}{l}\text { Aumenta la conectividad } \\
\text { estructural del paisaje }\end{array}$ & $\begin{array}{l}\text { Creando corredores locales donde pueden } \\
\text { habitar tanto fauna como flora }\end{array}$ & $\begin{array}{l}\text { México y } \\
\text { Costa Rica }\end{array}$ & $\begin{array}{l}\text { Estrada et al., 2000; León \& } \\
\text { Harvey, 2006; Medina et al., } 2007\end{array}$ \\
\hline Proveer ingresos adicionales & $\begin{array}{l}\text { Venta de madera para construcción o para } \\
\text { nuevas cercas vivas o la venta de fruta } \\
\text { proveniente de las cercas vivas }\end{array}$ & $\begin{array}{l}\text { Honduras, } \\
\text { Nicaragua y Brasil }\end{array}$ & $\begin{array}{l}\text { Chavarría et al., 2011; Queiroz et } \\
\text { al., } 2015 \text { Sousa et al., } 2016\end{array}$ \\
\hline $\begin{array}{l}\text { Reducción de la presión sobre } \\
\text { la vegetación remanente }\end{array}$ & $\begin{array}{l}\text { La presencia de cercas vivas en los predios } \\
\text { disminuye la necesidad de extraer madera } \\
\text { de los fragmentos de bosque remanentes }\end{array}$ & Costa Rica & Restrepo-Sáenz et al., 2004 \\
\hline $\begin{array}{l}\text { Capacidad de rebrote, auto } \\
\text { reparación y alta durabilidad }\end{array}$ & Se requiere poco trabajo de mantenimiento & Brasil & $\begin{array}{l}\text { Ayuk, 1997; } \\
\text { Nascimento et al., } 2009\end{array}$ \\
\hline $\begin{array}{l}\text { Actividad de bajo costo de } \\
\text { realizar }\end{array}$ & $\begin{array}{l}\text { Son consideradas de bajo costo, el } \\
\text { mantenimiento es más sostenible } \\
\text { económicamente que el costo de } \\
\text { mantenimiento de cercas muertas }\end{array}$ & $\begin{array}{l}\text { Costa Rica, } \\
\text { Nicaragua y Brasil }\end{array}$ & $\begin{array}{l}\text { Ayuk, } 1997 \text {; Torres et al., 2008; } \\
\text { Harvey et al., 2003; Queiroz et } \\
\text { al., } 2015\end{array}$ \\
\hline Provisión de sombra & $\begin{array}{l}\text { Reduce el estrés del ganado, aumentando la } \\
\text { producción de carne o producción lechera }\end{array}$ & $\begin{array}{l}\text { Costa Rica y } \\
\text { Nicaragua }\end{array}$ & Harvey et al., 2003 \\
\hline Controladoras de plagas & $\begin{array}{l}\text { Afecta el microclima del huerto de mango } \\
\text { afectando la distribución de los insectos } \\
\text { plagas }\end{array}$ & Senegal & Grechi et al., 2013 \\
\hline Mejorar la fertilidad del suelo & $\begin{array}{l}\text { Mitiga la pérdida de nutrientes del suelo, } \\
\text { devolviendo algunos nutrientes por medio } \\
\text { de la caída de hojarasca }\end{array}$ & $\begin{array}{l}\text { Nigeria, Kenia y } \\
\text { México }\end{array}$ & $\begin{array}{l}\text { Hulugalle \& Kang, } 1990 \text { Kiepe, } \\
\text { 1996, McIntyre et al., 1996, Peter } \\
\text { \& Lehmann, 2000, Olasantan,2000, } \\
\text { Kinama et al., 2005; Villanueva- } \\
\text { López et al., } 2015\end{array}$ \\
\hline \multicolumn{4}{|c|}{ Percepciones negativas } \\
\hline Podar o reparar las cercas & Requiere trabajo adicional & Honduras & Pérez et al., 2011 \\
\hline Remover o reubicar & $\begin{array}{l}\text { Resulta difícil cambiar el tamaño de los } \\
\text { potreros y los sistemas de rotación }\end{array}$ & $\begin{array}{l}\text { Costa Rica, } \\
\text { Honduras y México }\end{array}$ & $\begin{array}{l}\text { Somarriba, 1995, Harvey et al., } \\
\text { 2005, Pérez et al., 2011, Ruiz- } \\
\text { Guerra et al., } 2014\end{array}$ \\
\hline $\begin{array}{l}\text { Sombra a los sistemas } \\
\text { productivos }\end{array}$ & $\begin{array}{l}\text { Disminuye la productividad de los pastos } \\
\text { por el efecto de la sombra }\end{array}$ & Costa Rica & $\begin{array}{l}\text { Muñoz et al., 2003; } \\
\text { Nascimento et al., } 2009\end{array}$ \\
\hline Falta de información & $\begin{array}{l}\text { Desconocimiento de las bondades de las } \\
\text { cercas vivas }\end{array}$ & Brasil & Nascimento et al., 2009 \\
\hline Gasto económico & $\begin{array}{l}\text { Cambiarlas de sitio implica un gasto } \\
\text { económico que los agricultores no están } \\
\text { dispuesto a asumir }\end{array}$ & Burkina Faso & Ayuk, 1997 \\
\hline $\begin{array}{l}\text { Conflictos de intereses por la } \\
\text { plantación de cercas vivas }\end{array}$ & $\begin{array}{l}\text { Plantar árboles tiene significado de tenencia } \\
\text { de tierra y alinderación de propiedad }\end{array}$ & Malí & Levasseur et al., 2004 \\
\hline Dificultad de manejo & Espinas peligrosas para los niños o animales & & \\
\hline
\end{tabular}



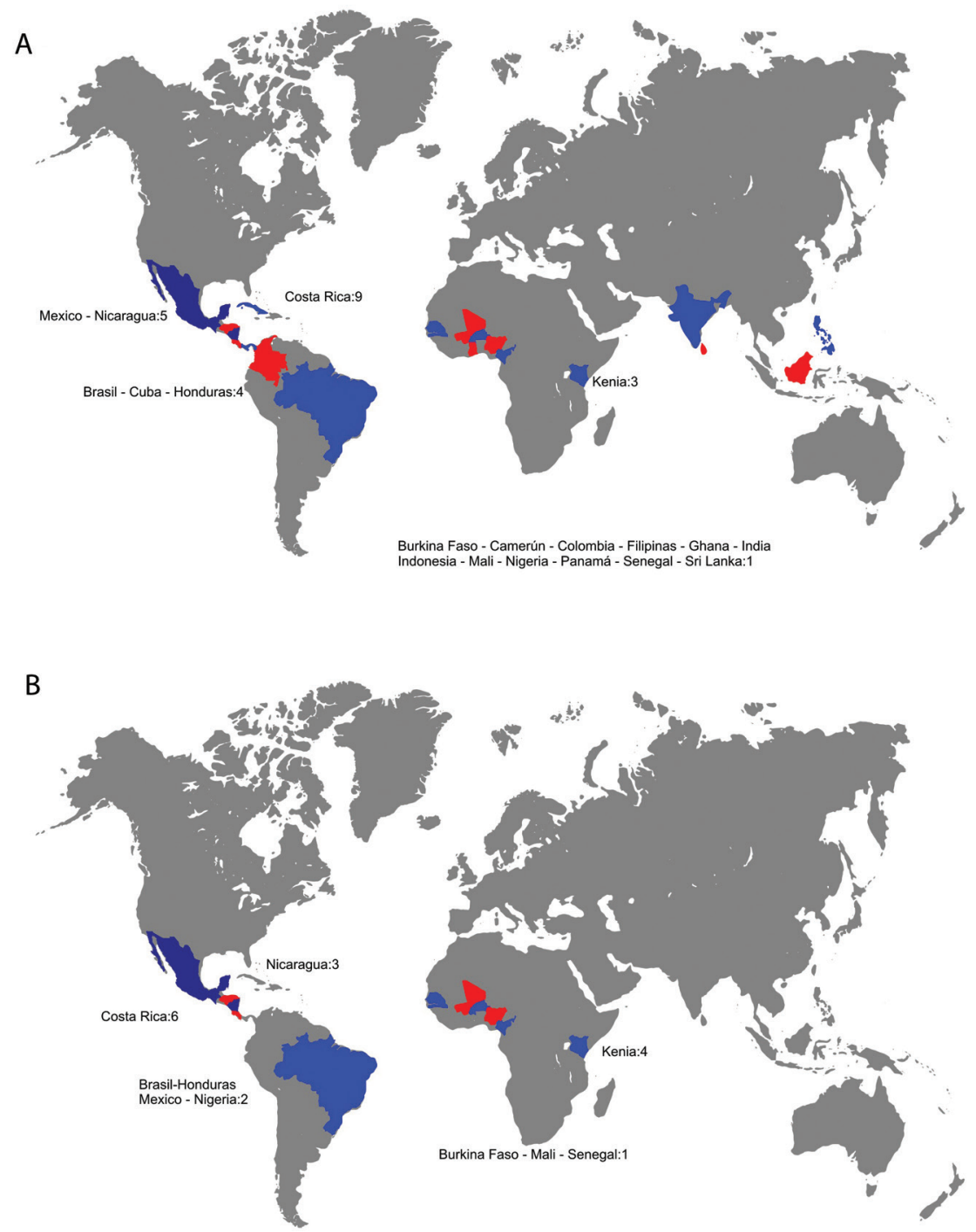

Fig. 1. Países con publicaciones sobre (A) usos que le dan los pobladores locales a las cercas vivas y (B) percepciones positivas o negativas sobre las cercas vivas en los paisajes rurales tropicales.

Fig. 1. Countries with publications on (A) uses that local Settlers give to live fences and (B) positive or negative perceptions about live fences in tropical rural landscapes.

Ruiz, \& Gutiérrez, 2002b; Alonso et al., 2002a; Harvey et al., 2003; Harvey et al., 2005).

Provisión de madera: La extracción de madera fue descrita en el $18 \%$ de los artículos en la región neotropical, seguido del $5 \%$ y el $1 \%$ de las regiones tropicales de África y Asia, respectivamente. Los artículos que reportaron este uso describen que intencionalmente los agricultores y ganaderos siembran especies maderables dentro de las cercas vivas (Erythrina berteroana, Samanea saman, Minquartia guianensis y Lysiloma divaricatum), para posteriormente aprovechar su madera (Romero, Chana, Montenegro, Sanchez, \& Guevara, 1991; Ayuk, 1997; Betancourt, 
Ibrahim, Harvey, \& Vargas, 2003; Muñoz, Harvey, Sinclair, Mora, \& Ibrahim, 2003; Villacis, Harvey, Ibrahim, \& Villanueva, 2003; Harvey et al., 2005; Zahawi, 2005; Love et al., 2009; Nascimento et al., 2009; Chavarría, Detlefsen, Ibrahim, Galloway, \& de Camino, 2011; Pérez et al., 2011; Egbe, Fonge, Mokake, Besong, \& Fongod, 2012; Grechi et al., 2013; Siles et al., 2013; Ruiz-Guerra, Rosas \& López-Acosta, 2014; Sousa, Detlefsen, Virginio, Tobar, \& Casanoves, 2016).

En la región tropical de África, las cercas vivas son establecidas con frecuencia para la extracción de postes debido a la poca disponibilidad de bosques cercanos; las especies más utilizadas son Guazuma ulmifolia y Gliricidia sepium; las cuales son de rápido crecimiento. En general, los agricultores de esta región están interesados en la plantación de cercas vivías con la participación de especies que sean resistentes a la sequía, que crezcan rápidamente y sean eficientes en la protección de sus campos de producción (Ayuk, 1997).

Provisión de leña: El aprovisionamiento de leña se encontró en el $20 \%$ de los artículos analizados de la región tropical. En la región tropical de Asia y África no se encontraron estudios sobre la extracción de leña de las cercas vivas, por lo cual no quiere decir que esta práctica no se realice en esta región, si no que no se reporta en artículos de revista científica. La extracción de leña es uno de los principales usos que los pobladores locales de la región tropical les dan a las cercas vivas, acompañados por la venta e intercambio por otros elementos importantes para sus fincas; dado que esta leña la utilizan como fuente de energía para la cocción de sus alimentos. Por ejemplo, en Centroamérica, conforman una de las principales fuentes de energía para el abastecimiento familiar, y las especies más utilizadas para este fin es el roble (Quercus sp.) y el guamo (Inga sp.). (Muñoz et al., 2003; Villacis et al., 2003; Otero \& Onaindia, 2009; Nascimento et al., 2009; Chavarría et al., 2011; Pérez et al., 2011; Siles et al., 2013; Ruiz-Guerra et al., 2014; Sousa et al., 2016).
Provisión de frutos: Este uso fue encontrado en el $9 \%$ de los artículos de la región neotropical, seguida del $6 \%$ de la región de África y el $1 \%$ de los artículos analizados para la región de Asia. Los frutos fueron utilizados para consumo humano o como alimento para el ganado (Olasantan, 2000; Reyes \& Rosado, 2000; Rowe et al., 2001; Harvey et al., 2003; Muñoz et al., 2003; Restrepo-Sáenz, Ibrahim, Harvey, Harmand, M., \& Morales, 2004; Kinama et al., 2005; Chavarría et al., 2011; Fogel, Mello, Fortuna, Neves, \& Sarmiento, 2012; Loyola et al., 2015). Algunas especies cuyos frutos son proveídos al ganado son el samán (Samanea saman), guanacaste (Enterolobium cyclocarpum), guayaba (Psidium guajava) y cítricos (Citrus spp.). Se encontró que en región de África tropical los agricultores realizan repoblamiento de las cercas vivas con árboles que puedan producir frutos (Cuadro 1), ya sea para complementar su dieta, proporcionar micro-nutrientes importantes a la familia; en algunos casos los agricultores venden la fruta en el mercado y obtienen un ingreso adicional (Abunyewa, Asiedu, Nyamekye, \& Cobbina, 2004). Algunos árboles frutales que son incorporados en los sistemas de cercas son el mango (Mangifera indica) que se ha expandido en África occidental y más específicamente en Senegal, donde se siembra para el consumo local y los mercados nacionales. Otros frutales encontrados en las cercas son la toronja (Citrus paradisi), mandarina (Citrus reticulata), naranja (Citrus sinensis), limón (Citrus limon), papaya (Carica papaya) y guayaba (Psidium guajava) las cuales son cosechadas en menor medida (Grechi et al., 2013).

Productos medicinales: La extracción de productos medicinales de las cercas vivas se reportó en el $1.2 \%$ de los artículos para el Neotrópico, seguido de la región tropical de África con $1.2 \%$, para la región tropical de Asia no se reportó este uso en el ámbito científico; lo cual no quiere decir que esta práctica de uso no se relice en esta región en particular. Por otra parte, en Brasil se producen medicinas caseras como el látex de Jatropha mollissima, el cual 
se usa para curar las heridas y enfermedades de la piel (Nascimento et al., 2009). En el trópico africano algunos árboles cultivados en las cercas como Acacia senegal se utiliza para elaborar ungüentos para dolores musculares. En Malí se extraen productos medicinales como el triturado de ramas de Lawsonia inermis para tratar principalmente el dolor de estómago y boca (Levasseur, Djimdé, \& Olivier, 2004). En África tropical, la mayoría de las plantas medicinales de las cercas vivas son plantadas por las mujeres, quienes tienen a cargo la labor de velar por la salud de la familia (Cuadro 1).

Provisión de servicios: En Colombia, México y Nigeria, las cercas vivas pueden estar compuestas de especies como Gliricidia sepium utilizadas para evitar la erosión y retener la humedad del suelo, las cuales favorecen la estabilidad de la temperatura del suelo (Otero \& Onaindia, 2009; Oshunsanya, 2013; Villanueva-López, Martínez-Zurimendi, Ramírez-Avilés, Aryal \& Casanova-Lugo, 2016). En países como Cuba las cercas vivas pasaron de usarse como barrera al ser utilizadas para proporcionar sombra a los animales vacunos y evitar el estrés (Fudora, Quintana, \& González, 2004).

En Asia tropical utilizan las cercas vivas para producir mayor biomasa, brindando un aporte de nutrientes a los cultivos, con esto se busca especialmente la fijación de nitrógeno, la especie más utilizada es aunque hay que advertir que no proporcionan la totalidad de nutrientes necesarios (Weerasinghe, 2007; Agus, Garrityb, \& Cassel, 1999).

En India utilizan las cercas vivas como rompevientos y para aumentar la retención de la humedad del suelo mediante la acumulación de la hojarasca (Cuadro 1). Además, tienen un rol importante en el control de la erosión de los suelos por medio de la disminución de la velocidad de escorrentía en los sistemas productivos; ya que la erosión reiterada por escorrentía impide la formación de humus estable en el suelo (Adhikary, Hombegowda, Barman, Jakhar, \& Madhu, 2016).

\section{Percepciones de las cercas vivas en los paisajes rurales tropicales}

Los resultados de las percepciones de las cercas vivas en la región tropical se presentan a continuación, y se clasifican de manera separada las percepciones positivas de las percepciones negativas de toda la región tropical.

Percepciones positivas de las cercas vivas. Los estudios revisados indican que los agricultores en la región tropical tienen percepciones variadas de las cercas vivas, desde el aumento de la productividad del predio hasta el aprovisionamiento de bienes y servicios (Maldonado et al., 2008). En Colombia y Costa Rica se encontraron similitudes en su percepción, allí los agricultores enuncian que con la plantación de cercas vivas en sus predios fomentan el aumento en la conectividad del paisaje, donde en los corredores locales pueden habitar tanto fauna como flora, lo anterior es visto por los agricultores como algo positivo para la conservación de la biodiversidad nativa (Estrada, Pierluigi, \& Rosamond, 2000; León \& Harvey, 2006; Medina, Harvey, Merlo, Vílchez, \& Hernández, 2007). Por otra parte, se identificó que perciben las cercas como un instrumento que puede proveer ingresos adicionales (Cuadro 2), como por ejemplo en la venta de madera, ya sea para construcción o para establecer nuevas cercas vivas (Chavarría et al., 2011; Abas et al., 2015; Queiroz, Maneschy, \& Marques, 2015; Sousa et al., 2016).

Otro aspecto positivo de la percepción sobre las cercas en la región neotropical es la disminución de la presión sobre la vegetación remanente, debido a que la presencia de las cercas vivas en los predios evita la necesidad de extraer madera de los fragmentos de bosque (Ayuk, 1997; Restrepo-Sáenz et al., 2004). Igualmente, otro componente que estimula el establecimiento de cercas vivas son los altos costos en la obtención de madera en el mercado, lo cual podría obtener beneficios importantes para la conservación de la biodiversidad (Harvey et al., 2005; Nascimento et al., 2009; Otero \& Onaindia, 2009; Hermuche et al., 2013; Loyola et al., 2015; Sousa et al., 2016). 
En Brasil, las percepciones sobre las cercas vivas reflejan aspectos positivos frente a las cercas muertas, puesto que la mayoría de los agricultores y ganaderos revelan la preferencia debido a su gran capacidad de rebrote, auto reparación y alta durabilidad (Nascimento et al., 2009). En Centroamérica se identificó la auto sostenibilidad de la cerca como un aspecto positivo a tener en cuenta, ya que se extraen estacas nuevas a partir de las cercas vivas existentes, lo cual hace que la renovación de cercas vivas sea considerada una actividad de bajo costo (Cuadro 2), creando la percepción que a largo plazo, el costo del mantenimiento sea más sostenible económicamente, lo cual contrasta con el costo de mantenimiento de cercas muertas (Harvey et al., 2003; Torres, Castro, \& Grande, 2008; Queiroz et al., 2015). Torres et al. (2008), demostraron que, en el estado de Veracruz México, los agricultores expresaron que construir cercas con postes de concreto y alambre de púas es la opción más costosa en comparación con plantar cercas vivas con especies nativas.

En la región neotropical, el conocimiento de los ganaderos por la provisión de sombra es de carácter positivo (Cuadro 2). La provisión de sombra en estos sistemas productivos es de vital importancia debido a que esta reduce el estrés que ocasiona el calor, proveyendo un ambiente más confortable y facilita las tasas reproductivas, y ganancia de peso y producción de leche en el ganado (Harvey et al., 2003). Por lo general, los agricultores y ganaderos manifiestan que ven de buena manera las cercas vivas, por proveer productos y servicios; dado que son establecidas de acuerdo con motivaciones de mejoramiento de producción y no considerando motivos de conservación (Pérez et al., 2011; Villanueva-López et al., 2016).

Por otro lado, actualmente el establecimiento de cercas vivas en África tropical se ha hecho popular entre los agricultores, pues perciben beneficios en el desarrollo de los sistemas productivos. Una de las percepciones positivas que mencionan los agricultores es la durabilidad de las cercas vivas, debido a que una vez establecidas, el trabajo adicional es menor; asimismo, las cercas son asociadas con funciones diferentes a la de protección y delimitación de predios, tales como el control de la erosión y la protección contra el viento (Ayuk, 1997). Al mismo tiempo se identificó que las cercas vivas podrían actuar como controladoras de plagas, en vista de que puede afectar la distribución espacial de la comunidad de artrópodos en los cultivos, reduciendo las poblaciones de polillas y la infestación por las moscas de la fruta en huertos de mango (Cuadro 2) (Grechi et al., 2013).

Algunos de los artículos de la región tropical africana y el Neotrópico consultados, indican que las cercas vivas logran mejorar la fertilidad del suelo (Cuadro 2), siempre y cuando las raíces de los árboles que componen la cerca, estén más profundas en el suelo que las raíces de los cultivos; para disminuir la competencia por debajo del suelo y obteniendo un efecto positivo en los rendimientos de los cultivos (Olasantan, 2000; Peter \& Lehmann, 2000). Los agricultores manifiestan que otro beneficio es poder mitigar la pérdida de nutrientes del suelo (Kinama et al., 2005), debido a que las cercas vivas pueden aumentar la traspiración y aumentar las tasas de infiltración de agua lluvia (McIntyre, Riha, \& Ong, 1996) restituyendo algunos nutrientes al suelo por medio de la caída de hojarasca (Cuadro 2) (Hulugalle \& Kang, 1990; Kiepe, 1996; Gómez et al., 2008; Loyola et al., 2014; VillanuevaLópez et al., 2014, 2015, 2016).

En Asia tropical, los agricultores adoptan las cercas vivas esencialmente porque perciben servicios que les favorecen sobre los rendimientos de los sistemas productivos, incluso no se preocupan por la disposición o técnica de plantación de las cercas vivas, sino que se preocupan por la generación de dividendos a corto plazo (Agus et al., 1999). Es importante destacar que los agricultores de esta región tropical, no tienen en cuenta la conservación de la biodiversidad en la instalación de cercas vivas, sino que buscan aportar en mayor medida los nutrientes importantes del suelo como lo pueden ser el nitrógeno, fosforo y especialmente 
carbono orgánico (Rowe et al., 2001; Weerasinghe, 2007; Adhikary et al., 2016).

Percepciones negativas de las cercas vivas. -Uno de los aspectos que impide el establecimiento de cercas vivas en las regiones tropicales es la necesidad de podar o reparar las cercas, ya que puede requerir de considerable trabajo adicional (Pérez et al., 2011). Otro aspecto identificado, es el hecho de que una vez que las cercas vivas son plantadas, resultan difíciles de remover y reubicar, lo que dificulta cambiar el tamaño de los potreros o de los sistemas de rotación (Somarriba, 1995; Levasseur et al., 2004; Harvey et al., 2005; Pérez et al., 2011; Ruiz-Guerra et al., 2014).

También se consideró la sombra como una posible desventaja, dado que un exceso de sombra, puede disminuir la productividad de los pastos y, por lo tanto, reducir el área productiva general de la finca (Cuadro 2) (Muñoz et al., 2003). Así, los agricultores manifestaron que por lo general impiden que las cercas vivas crezcan demasiado y para esto realizan podas para controlar el tamaño de la copa de los árboles (Somarriba, 1995; Harvey et al., 2005). Igualmente, un estudio realizado en el estado de Pernambuco Brasil, demuestra que una percepción negativa es el excesivo trabajo que se debe realizar como reparaciones, podas y reubicaciones, lo que conlleva a implementar cercas muertas debido a la facilidad de construcción y al poco mantenimiento, el cual representa menos trabajo que puede ser invertido en otras actividades que demanda el sistema productivo (Nascimento et al., 2009).

Una razón por la cual las cercas vivas dejan de establecerse en la región neotropical es la falta de información de las funciones que pueden brindar, ya sean ecológicas o simplemente beneficios que puede traer al sistema productivo (Cuadro 2). Así ocurre en Brasil, donde los agricultores manifestaron que una de las razones para no implementar cercas vivas es la falta de información y total desconocimiento de las bondades de las cercas vivas sobre las cercas muertas (Nascimento et al., 2009). El problema de desconocimiento de las cercas vivas lo atribuyen a la falta de apoyo del gobierno y sus políticas rurales. En Nicaragua y Honduras, los agricultores manifestaban no realizar poda o adelgazamiento de las cercas vivas debido a la falta de conocimientos, lo que ocasiona un mal manejo, lo que ocasiona un desaprovechamiento de sus beneficios para los agricultores y los sistemas productivos (Sousa et al. 2016).

Un caso particular se da en África tropical donde los agricultores manifestaron dificultad al momento de manejar las cercas vivas, debido al peligro que representan las espinas para los niños o animales de corral (Levasseur et al., 2004). Estudios en Burkina Faso revelan que la probabilidad de implementar cercas tiene una relación negativa si el agricultor utiliza cercas muertas, ya que las cercas muertas son herramientas alternativas y cambiar surgiría un gasto económico que los agricultores no están dispuesto a asumir; lo anterior sirve como indicador de riqueza y sugiere que los agricultores que utilizan cercas vivas se encuentran económicamente estables (Ayuk, 1997).

En África Occidental se generan conflictos de intereses por la implantación de cercas vivas, dado que plantar un árbol tiene significado de tenencia de tierra y alinderación de propiedad. Este conflicto afecta directamente la plantación de cercas vivas ya que, por cuestión legal no se permite a los arrendatarios plantar árboles ni mucho menos establecer cercas vivas, ya que esto podría traer problemas legales al momento de heredar. Por lo tanto, en la mayoría de los casos las cercas vivas no surgirían y tendrían que implementar métodos alternos como el caso de cercas muertas (Levasseur et al., 2004). En general, el uso de cercas vivas en los paisajes agropecuarios tropicales podría aumentar la productividad del predio lo que podría promover la conservación de los bosques o parches remanentes que aún persisten en la región.

En conclusión, las cercas vivas, además, de servir para delimitar predios y potreros traen beneficios sobre los paisajes rurales donde se implementan, ofreciendo beneficios a los sistemas productivos que encadenan aumentos en la productividad final de la finca (Maldonado et al. 2008). Por otra parte, en las tres regiones 
tropicales se encontró que las cercas vivas se presentan como un componente importante en el mejoramiento de las condiciones de los suelos agrícolas y ganaderos (Otero \& Onaindia, 2009; Oshunsanya, 2013; Villanueva-López et al., 2016).

De acuerdo con la literatura analizada encontramos que las cercas vivas son implementadas teniendo en cuenta sus múltiples beneficios, lo que comprueba la hipótesis planteada y se demuestra que la utilización de las cercas vivas en la región tropical se realiza, ya sea para el suministro de bienes y servicios ambientales como para proveer beneficios a sus sistemas productivos.

Por otra parte, se confirmó la hipótesis que los agricultores y ganaderos tienen apreciaciones a favor y en contra sobre las cercas vivas, acerca de su plantación y mantenimiento dentro de sus sistemas productivos. Se encontró que estas afirmaciones se producían de acuerdo con sus experiencias vividas en sus predios o a la precaria información que recibían sobre ellas. Es de señalar que las percepciones positivas declaradas fueron teniendo en cuenta los recursos que las cercas proveen a los agricultores y que las percepciones negativas tenían como sustento las desventajas que podría ocasionar al rendimiento de los sistemas productivos.

Como resultado de la elaboración de esta revisión de literatura científica surgen nuevas perspectivas a futuro como evaluar el efecto de la presencia de diferentes estratos verticales en cuanto a la estructura (DAP y dosel) de los árboles que componen las cercas vivas, lo cual podría mejorar la habitabilidad de fauna y flora en el paisaje rural tropical. Conjuntamente, se sugiere investigar sobre los efectos que pueden causar en la fauna las cercas vivas con presencia de elementos como grandes rocas, tocones o troncos que podrían suministrar refugio, sobre todo a invertebrados.

Por otro lado, se recomienda realizar investigación a las cercas vivas desde el enfoque de la ecología de poblaciones e interacciones bióticas que se puedan presentar y conjuntamente desde la dimensión social.
Consideramos que se debe profundizar a cerca de los beneficios que producen las cercas vivas sobre los rendimientos de los sistemas agropecuarios de la región rural tropical, facilitando no solamente resolver las incertidumbres de los agricultores y ganaderos sobre el establecimiento de cercas vivas, sino que propiciar la conservación de la fauna y flora nativa que aún persiste en estas áreas fragmentadas de los paisajes rurales tropicales.

Por último, lo que se busca con esta revisión bibliográfica es generar iniciativas que vayan hacia la formulación de políticas rurales, la creación de programas de estímulo al establecimiento de cercas vivas ya sea por medio de incentivos como pagos por servicios ambientales (PSA) o indultos tributarios y brindar información a instituciones, agricultores y ganaderos de la región rural tropical.

\section{AGRADECIMIENTOS}

Expresamos nuestra entera gratitud a Nicolás Urbina por su contribución en la revisión y valiosos aportes para la consolidación del documento, a la Pontificia universidad Javeriana por proveer acceso a las bases de datos bibliográficas en las cuales se encontraron la mayoría de los artículos utilizados para esta revisión. También agradecemos a todos los amigos y familiares que de alguna manera nos brindaron su apoyo y motivación.

\section{RESUMEN}

La expansión de la producción agrícola y ganadera ha sido un factor clave en la deforestación en el mundo y especialmente en la región tropical. En consecuencia, más de la mitad de la superficie terrestre del planeta se utiliza para el establecimiento de sistemas productivos, en este contexto las cercas vivas pueden generan efectos positivos sobre el ambiente y proveer bienes y servicios ambientales. En este trabajo hacemos una revisión sobre los efectos que tienen las cercas vivas sobre los sistemas productivos, analizando los usos y percepciones que tienen los agricultores. Esta revisión abarca las diferentes regiones tropicales del mundo. Se encontró que las cercas vivas estuvieron principalmente vinculadas a la delimitación de predios y potreros. No obstante, sus usos y beneficios son múltiples, entre los que se encontraron suministrar forraje, extracción 
de madera, proveer frutos, servir como rompevientos, generar medicinas, evitar la erosión del suelo, retener la humedad y suministrar aportes nutricionales. Además, se encontraron percepciones a favor como aumentar la conectividad del paisaje, aumentar los ingresos económicos, reducir los costos productivos, reducir la presión sobre la vegetación remanente, tener bajo costos de mantenimiento, contribuir al control de plagas y mejoran la fertilidad. Sin embargo, también se encontraron percepciones negativas como el excesivo trabajo al repararlas o podarlas, la sombra puede afectar la producción agrícola, la falta de información desestimula su establecimiento, costos elevados de establecimiento, generación de conflictos de propiedades y dificultad de manejo. Se encontró que los usos por parte de los agricultores están enfocados a mejorar los rendimientos de los sistemas productivos, pero también a la conservación de recursos naturales como el suelo y la biodiversidad. Se proponen temas de investigación para el futuro como determinar los criterios en la elección de especies nativas para establecer y enriquecer las cercas vivas dando valor a aspectos ecológicos y socioeconómicos, además, se debe profundizar en los beneficios que producen sobre los rendimientos de los sistemas productivos Se sugiere realizar estudios sobre las cercas vivas en la región tropical de Australasia debido a que no se encontraron estudios en esta región, así se podría conocer el estado de su biodiversidad y servicios que brinda a la comunidad rural. Por último, lo que se busca con esta revisión es generar iniciativas que fomenten la formulación de políticas rurales, la creación de programas que estimulen el establecimiento de cercas vivas, ya sea por medio de pagos por servicios ambientales, compensaciones tributarias u otro tipo de mecanismos. Proveer información adecuada a agricultores y ganaderos es un aspecto central de dinamiza el establecimiento de las cercas vivas.

Palabras clave: cercas vivas, biodiversidad tropical, sistema silvopastoril, sistemas agropecuarios, agroforestería.

\section{REFERENCIAS}

Abas, E. L., Corpuz, O. S., \& Cabilo, L. D. (2015). Light Regime Under Eucalyptus deglupta as Hedgerows and its Effect on Intercropped Zea mays. American Journal of Agriculture and Forestry, 3, 19-29.

Abunyewa, A., Asiedu, E. K., Nyamekye, A. L., \& Cobbina, J. (2004). Alley cropping Gliricidia sepium with Maize: 1. The Effect of Hedgerow Spacing, Pruning Height and Phosphorus Application Rate on Maize Yield. Journal of biological Sciences, 4, 81-86.

Adhikary, P. P., Hombegowda, H. C., Barman, D., Jakhar, P., \& Madhu, M. (2016). Soil erosion control and carbon sequestration in shifting cultivated degraded highlands of eastern India: performance of two contour hedgerow systems. Agroforestry Systems, $158,31-40$.
Agus, F., Garrityb, D. P., \& Cassel, D. K. (1999). Soil fertility in contour hedgerow systems on sloping oxisols in Mindanao, Philippines. Soil \& Tillage Research, 50, 159-167.

Alonso, J., Febles, G., Ruiz, T. E., \& Gutiérrez, J. C. (2002). Efecto de la fase lunar en el establecimiento de piñón florido (Gliricidia sepium) como cerca viva. Revista Cubana de Ciencia Agrícola, 2, 187-191.

Alonso, J., Febles, G., Ruiz, T. E., Torres, V., \& Gutiérrez, J. C. (2002). Establecimiento del piñón florido (Gliricidia sepium) como cerca viva bajo diferentes condiciones de explotación ganadera. Revista Cubana de Ciencia Agrícola, 36, 293-302.

Ayuk, E. T. (1997). Adoption of Agroforestry Technology: The Case of Live Hedges in the Central Plateau of Burkina Faso. Agricultural Systems, 54, 189-206.

Barbier, E. B., \& Burgess, J. C. (2001). The economics of tropical deforestation. Journal of Economic Surveys, $15,413-432$.

Betancourt, K., Ibrahim, M., Harvey, C. A., \& Vargas, B. (2003). Efecto de la cobertura arbórea sobre el comportamiento animal en fincas ganaderas de doble propósito en Matiguás, Matagalpa, Nicaragua. Agroforestería en las Américas, 10, 47-51.

Budowski, G. (1993). The scope and potential of agroforestry in Central America. Agroforestry Systems, 23, 121-131.

Burel, F. (1996). Hedgerows and their role in agricultural landscapes. Critical Reviews in Plant Sciences, 15, 169-190.

Calle, Z., Murgueitio, E., \& Chará, J. (2012). Integrating forestry, sustainable cattle-ranching and landscape restoration. Unasylva, 63, 31-40.

Cárdenas, G., Harvey, C. A., Ibrahim, M., \& Finegan, B. (2003). Diversidad y riqueza de aves en diferentes hábitats en un paisaje fragmentado en Cañas, Costa Rica. Agroforestería en las Américas, $10,39-40$.

Chavarría, A., Detlefsen, G., Ibrahim, M., Galloway, G., \& de Camino, R. (2011). Análisis de la productividad y la contribución financiera del componente arbóreo en pequeñas y medianas fincas ganaderas de la subcuenca del río Copán, Honduras. Agroforestería en las Américas, 48, 146-156.

Cubbage, F., Balmelli, G., Bussoni, A., Noellemeyer, E., Pachas, A., Fassola, H., Colcombet, L., Rossner, B., Frey, G., Dube, F., de Silva, M., Stevenson, H., Hamilton, J., \& Hubbard, W. (2012). Comparing silvopastoral systems and prospects in eight regions of the world. Agroforestry Systems, 86, 303-314.

de Castro, G. C., \& van den Berg, E. (2013). Structure and conservation value of high-diversity hedgerows in 
southeastern Brazil. Biodiversity and Conservation, 22, 2041-2056.

Egbe, E. A., Fonge, B. A., Mokake, S., Besong, M., \& Fongod, A. (2012). The effects of green manure and NPK fertilizer on the growth and yield of maize (Zea mays $\mathrm{L})$ in the mount Cameroon region. Agriculture and Biology Journal of North America, 3, 82-92.

Estrada, A., Pierluigi, C., \& Rosamond, C. E. (2000). Bird species richness in vegetation fences and in strips of residual rain forest vegetation at Los Tuxtlas, Mexico. Biodiversity and conservation, 9, 1399-1416.

Etongo, D., Djenontin, I. N. S., Kanninen, M., Fobissie, K., Korhonen-Kurki, K., \& Djoudi, H. (2015). Land tenure, asset heterogeneity and deforestation in Southern Burkina Faso. Forest Policy and Economics, 61, 51-58.

FAO. 2016. El Estado de los bosques del mundo 2016. Los bosques y la agricultura: desafíos y oportunidades en relación con el uso de la tierra. Roma.

Fogel, J. F., Mello, G. A., Fortuna, G. C., Neves, H. H., \& Sarmiento, L. C. E. (2012). Moirão-vivo para o sistema de condução do maracujazeiro-amarelo. Cadernos de Agroecologia, 7, 1-4.

Foley, J. A., Defries, R., Asner, G. P., Barford, C., Bonan, G., Carpenter, S. R., ... Snyder, P. K. (2005). Global Consequences of Land Use. Science, 309, 570-574.

Fudora, O., Quintana, F. O., \& González, M. E. (2004). A note on dehorning of river buffaloes as an alternative for decreasing the deterioration of live fences and tres. Cuban Journal of Agricultural Science, 38, $45-48$.

Fuentealba, B. D., \& Martínez-Ramos, M. (2014). Transplanting native tree seedlings to enrich tropical live fences: an ecological and socio-economic analysis. Agroforestry Systems, 88, 221-236.

Garner, W., \& Steinberger, Y. (1989). A proposed mechanism for the formation of "fertile islands" in the desert ecosystem. Journal of Arid Environments, 16, 257-262.

Grechi, I., Sane, B. C. A., Diame, L., De Bon, H., Benneveau, A., Michels, T., . . . Rey, J.-Y. (2013). Mangobased orchards in Senegal: diversity of design and management patterns. Fruits, 68, 447-466.

Gómez, I., Olivera, Y., Botello, A., \& Espinosa, R. (2008) Efecto del marco de siembra en la producción de postes vivos de Gliricidia sepium. Pastos y Forrajes, $31(4), 1$.

Haddad, N. M., Brudvig, L. A., Clobert, J. Davies, K. F., Gonzalez, A., Holt, R. D., ... Townshend, J. R. (2015). Habitat fragmentation and its lasting impact on Earth's ecosystems. Science Advances, l(2), e1500052.
Harvey, C. A., Villanueva, C., Villacis, J., Chacón, M., Muñoz, D., López, M., ... Sinclair, F. L. (2003). Contribución de las cercas vivas a la productividad e integridad ecológica de los paisajes agrícolas en América Central. Agroforestería en las Américas, 10, 30-39.

Harvey, C. A., Tucker, N., \& Estrada, A. (2004). Live fences, isolated trees and windbreaks: tools for conserving biodiversity in fragmented tropical landscapes? Washington, DC, USA: Island Press.

Harvey, C. A., Villanueva, C., Villacís, J., Chacón, M., Muñoz, D., López, M., . . . Sinclair, F. L. (2005). Contribution of live fences to the ecological integrity of agricultural landscapes. Agriculture, Ecosystems \& Environment, 111(1-4), 200-230.

Hermuche, P. M., Vieira, M. D. L., \& Sano, E. E. (2013). Modeling tree cover changes in a pasture-dominated landscape by adopting silvopastoral practices in a dry forest region in Central Brazil. Agroforestry System, $87,881-890$.

Horne, P. M., \& Stür, W. W. (1999). Developing forage technologies with smallholder farmers - how to select the best varieties to offer farmers in Southeast Asia. ACIAR Monograph, 62-80.

Hulugalle, N. R., \& Kang, B. T. (1990). Effect of Hergerow species in alley Cropping Systems on Surface Soil Physical Properties of an Oxic Paleustalf in South western Nigeria. Journal of Agricultural Science, 114, 301-307.

Kiepe, P. (1996). Cover and barrier effect of Cassia siamea hedgerows on soil conservation in semi-arid Kenya. Soil Technology, 9, 161-171.

Kinama, J. M., Stigter, C. J., Ong, C. K., Ng’ang’a, J. K., \& Gichuki, F. N. (2005). Evaporation from soils below sparse crops in contour hedgerow agroforestry in semi-arid Kenya. Agricultural and Forest Meteorology, 130, 149-162.

Levasseur, V., Djimdé, M., \& Olivier, A. (2004). Live fences in Ségou, Mali: an evaluation by their early users. Agroforestry Systems, 60, 131-136.

León, M. C., \& Harvey, C. A. (2006). Live fences and landscape connectivity in a neotropical agricultural landscape. Agroforestry Systems, 68(1), 15-26.

Love, B. E., Bork, E. W., \& Spaner, D. (2009). Tree seedling establishment in living fences: a low-cost agroforestry management practice for the tropics. Agroforestry Systems, 77, 1-8.

Loyola, H. O., Pérez, C. I., Triana, G. D., Valido, T. A., \& Yeró, P. I. (2014). Agroproductive Evaluation of Moringa oleifera Lam in Hedges, under Edafo-climatic Conditions. Revista de Producción Animal, 26(2).

Loyola, H. O., Valido, T. A., Triana, G. D., Pérez, C. I., Yero, P. I., \& Gonzáles, G. D. C. (2015). Evaluación 
de la retención de carbono y la fauna edáfica en asocio con Moringa oleifera Lam en cercas vivas. Centro Agrícola, 42, 75-81.

Maldonado, M. N., Grande, D. J., Fuentes, E. E., Hernández, S., Pérez-Gil, F., \& Gómez, A. (2008). Los sistemas silvopastoriles de la región tropical húmeda de México: El caso de Tabasco. Zootecnia Tropical, 26, 305-308.

McIntyre, B. D., Riha, S. J., \& Ong, C. K. (1996). Light interception and evapotranspiration in hedgerow agroforestry systems. Agricultural and Forest Meteorology, 81, 31-40.

Medina, A., Harvey, C. A., Merlo, D. S., Vílchez, S., \& Hernández, B. (2007). Bat Diversity and Movement in an Agricultural Landscape in Matiguás, Nicaragua. Biotropica, 39, 120-128.

Muñoz, D., Harvey, C. A., Sinclair, F. L., Mora, J., \& Ibrahim, M. (2003). Conocimiento local de la cobertura arbórea en sistemas de producción ganadera en dos localidades de Costa Rica. Agroforestería en las Américas, 10, 61-68.

Murgueitio, E., Cuartas, C., \& Naranjo, J. F. (2008). Ganadería del Futuro: Investigación Para el Desarrollo. Fundación CIPAV, Cali, Colombia.

Murgueitio, E., Calle, Z., Uribe, F., Calle, A., \& Solorio, B. (2011). Native trees and shrubs for the productive rehabilitation of tropical cattle ranching lands. Forest Ecology and Management, 261, 1654-1663.

Nascimento, V. T., Sousa, L. G., Alves, A. G. C., Araújo, E. L., \& Albuquerque, U. P. (2009). Rural fences in agricultural landscapes and their conservation role in an area of caatinga (dryland vegetation) in Northeast Brazil. Environment, Development and Sustainability, 11, 1005-1029.

Olasantan, F. O. (2000). Effect of nitrogen rate on okra and tomato in Gliricidia alley cropping system in Southwestern Nigeria.Tropical Agricultural Research and Extension, 3, 110-114.

Oshunsanya, S. O. (2013). Spacing effects of vetiver grass (Vetiveria nigritana Stapf) hedgerows on soil accumulation and yields of maize-cassava intercropping system in Southwest Nigeria. Catena, 104, 120-126.

Otero, J., \& Onaindia, M. (2009). Landscape structure and live fences in Andes Colombian agrosystems: upper basin of the Cane-Iguaque River. Revista de Biologia Tropical, 57(4), 1183-1192.

Peter, I., \& Lehmann, J. (2000). Pruning effects on root distribution and nutrient dynamics in an acacia hedgerow planting in northern Kenya. Agroforestry Systems, 50, 59-75.

Pereira, H. M., Leadley, P. W., Proença, V., Alkemade, R., Scharlemann, J. P. W., Fernandez-Manjarrés, J. F., ...
Walpole, M. (2010). Scenarios for Global Biodiversity in the 21st Century. Science, 330, 1496-1501.

Pérez, E., Richers, B., DeClerck, F., Casanoves, F., Gobbi, J., \& Benjamin, T. (2011). Uso y manejo de la cobertura arbórea en sistemas silvopastoriles en la subcuenca del río Copán, Honduras. Agroforestería en las Américas, 48, 26-35.

Pulido-Santacruz, P., \& Renjifo, L. M. (2010). Live fences as tools for biodiversity conservation: a study case with birds and plants. Agroforestry Systems, 81, 15-30. ISSN 0167-4366

Queiroz, J. F., Maneschy, R. Q., \& Marques, M. N. C. (2015). Modelos econômicos de sistemas silvipastoris com cercas vivas. Cadernos de Agroecologia, $10(3)$.

Restrepo-Sáenz, C., Ibrahim, M., Harvey, C., Harmand, J. M., \& Morales, J. (2004). Relaciones entre la cobertura arbórea en potreros y la producción bovina en fincas ganaderas en el trópico seco en Cañas, Costa Rica. Agroforestería en las Américas, 41, 29-36.

Reyes, S. A., \& Rosado, I. A. (2000). Plantas utilizadas como cercas vivas en el estado de Veracruz. Madera y Bosques, 6, 55-71.

Romero, F., Chana, C., Montenegro, J., Sanchez, L. A., \& Guevara, G. (1991). Productividad de Gliricidia sepium y Erythrina berteroana en Cercas Vivas Manejadas Bajo Tres Frecuencias de Poda en la Zona Atlántica de Costa Rica. Agroforestería, 6.

Rowe, E. C., van Noordwijk, M., Suprayogo, D., Hairiah, K., Giller, K. E., \& Cadisch, G. (2001). Root distributions partially explain $\mathrm{N}$ uptake patterns in Gliricidia and Peltophorum hedgerow intercropping systems. Plant and Soil, 235, 167-179.

Ruiz-Guerra, B., López-Acosta, J. C., Zaldivar-Riverón, A., \& Velázquez-Rosas, N. (2015). Braconidae (Hymenoptera: Ichneumonoidea) abundance and richness in four types of land use and preserved rain forest in southern Mexico. Revista Mexicana de Biodiversidad, 86, 164-171.

Ruiz-Guerra, B., Rosas, N. V., \& López-Acosta, J. C. (2014). Plant Diversity in Live Fences and Pastures, Two Examples from the Mexican Humid Tropics. Environmental Management, 54, 656-667.

Siles, P., Rayo, M. J., Rugama, A. F., \& Molina, L. (2013). Diversidad arbórea en cercas vivas y dos fragmentos de bosque en la comunidad de Santa Adelaida, Estelí. Encuentro, 96, 60-76.

Somarriba, E. (1995). Guayaba en potreros: Establecimiento de Cercas vivas y Recuperación de Pasturas degradadas. Agroforestería en las Américas, 6, 27-29.

Sousa, K. F. D., Detlefsen, G., Virginio, E. M., Tobar, D. T., \& Casanoves, F. (2016). Timber yield from 
smallholder agroforestry systems in Nicaragua and Honduras. Agroforestry Systems, 90, 207-218.

Torres, R. J. A., Castro, R. F., \& Grande, D. C. (2008). Cercas de uso pecuario en la cuenca del río La Antigua, México: Inventario florístico y costo de construcción. Zootecnia Tropical, 26, 279-283.

Villacis, J., Harvey, C. A., Ibrahim, M., \& Villanueva, C. (2003). Relaciones entre la cobertura arbórea y el nivel de intensificación de las fincas ganaderas en Río Frío, Costa Rica. Agroforestería en las Américas, $10,17-23$.

Villanueva-López, G., Martínez-Zurimendi, P., CasanovaLugo, F., Ramírez-Avilés, L., \& Montañez-Escalante, P. I. (2015). Carbon storage in livestock systems with and without live fences of Gliricidia sepium in the humid tropics of México. Agroforestry Systems, 89, 1083-1096.

Villanueva-López, G., Martínez-Zurimendi, P., RamírezAvilés, L., Aryal, D. R., \& Casanova-Lugo, F. (2016) Live fences reduce the diurnal and seasonal fluctuations of soil $\mathrm{CO} 2$ emissions in livestock systems. Agronomy for Sustainable Development, 36(1), 1-8.
Villanueva-López, G., Martínez-Zurimendi, P., RamírezAvilés, L., Casanova-Lugo, F., \& Jarquín-Sánchez, A. (2014). Influence of livestock systems with live fences of Gliricidia sepium on several soil properties in Tabasco, Mexico. Ciencia e Investigación Agraria, 41(2), 175-186.

Weerasinghe, M. (2007). Variation in Soil Fertility and Capacity for Supplying Soil Nutrients in A Hedgerow Intercropping System with Different Tree Species in The Upcountry Wet Zone of Sri Lanka. Ceylon Journal of Science, 36, 65-79.

Wehling, S., \& Diekmann, M. (2009). Importance of hedgerows as habitat corridors for forest plants in agricultural landscapes. Biological Conservation, 142, 2522-2530.

Zahawi, R. A. (2005). Establishment and Growth of Living Fence Species: An Overlooked Tool for the Restoration of Degraded Areas in the Tropics. Restoration Ecology, 13, 92-102.

Zuria, I., Gates, J. E., \& Castellanos, I. (2007). Artificial nest predation in hedgerows and scrub forest in a human-dominated landscape of central Mexico. Acta Oecologica, 31, 158-167. 